\title{
Effective Project Management and the Role of Quality Assurance throughout the Project Life Cycle
}

\author{
Monier Madison Ouabira, Hengameh Fakhravar
}

\begin{abstract}
Quality is a fundamental requirement in effective project management. Effective project management entails a steady focus on quality management as well as achievement of all user requirements as defined during the requirements engineering phase of project implementation. Quality assurance must be executed throughout the project development cycle as a new normal in reducing errors and challenges during project development. Conducting quality assurance throughout the project development cycle has many benefits to both the project as well as the project development team. Understanding the research approach to use is critical in achieving high-quality findings in projects. There is a need to understand how to utilize deductive, inductive, and abductive research reasoning when conducting project implementation.
\end{abstract}

Index Terms - Assurance, Management, Project Management, Quality.

\section{INTRODUCTION}

Project management concepts and strategies are at the center of every engineering project and task. According to [1], the complexity and lack of similarity between engineering projects and tasks have caused challenges in identifying a universal approach to managing all projects. As such, engineering management is dependent on multiple quality assurance principles, theories, and models that must all be used to ensure that any engineering project or task achieves the intended user requirements and quality objectives. Quality is a core requirement for effective engineering management since quality is at the center of all engineering projects and systems [2]. Quality is a virtue in engineering design and management, and achieving highquality standards is mandatory for effective project implementation and execution.

Researchers must have an adept understanding of the differences, advantages, and limitations of the various research methodologies and approaches, mainly deductive, inductive, and abductive. The research approaches or methodologies form the main reasoning that guides the research processes, and thus the research outcome [3]. Understanding these concepts is important as it makes it easy for researchers to choose the best research approach for their select research question and problem. This report outlines the differences between the three core research approaches, outlining instances in which each one is desired. This report also outlines the core characteristics for each of the named

Submitted on January 21, 2021.

Published on July 29, 2021.

M. M. Ouabira, Ph.D. student in Engineering Management and Systems Engineering, Old Dominion University, USA.

(e-mail: mouab001@odu.edu) research approaches, the impact of quality on project management, and the need for quality assurance for project management.

Additionally, this study is going to examine the importance of research design and analyzing and coming up with a good research design matters a lot in project implementation and execution. A research design is an overall structure of a research project. The main reason why researchers construct a research design is to ensure that the information collected in the research specifically answers the initial question. A project with a poorly constructed research design is likely to fail since it tells more about the internal structuring of the whole project. By looking at a research design of a particular project, one is likely to tell of its outcomes. Since the research method is dependent on the type of research design, one is urged to do a critical analysis of the type of research design to use and make sure that it perfectly suits the entire project.

\section{PROBLEM STATEMENT}

The main objective of this research is how to explore the diversity of research methods, and what has not been researched is how to evaluate the role of quality assurance throughout a project life cycle. In addition, how to evaluate what effective project management requires and to explore research and how it should be conducted.

\section{EFFECTIVE PROJECT MANAGEMENT}

Effective project management requires a combination of various engineering principles, techniques, theories, and effective teamwork to succeed. According to [4], project success and project management success are two independent concepts and need to be evaluated and managed independently. Project success is the overall effectiveness of the project to meet user requirements and specifications, whereas project management success refers to the overall effectiveness in managing the various aspects related to the project [5]. Project success is defined by various elements as they relate to project management. These elements are referred to as project success criteria and include the quality of project management process, project delivery within the time limits, project delivery within budgets, user satisfaction, net project benefits, meeting specified project quality, meeting project stakeholder satisfaction, and meeting specified project service quality. Projects that meet these

H. Fakhravar, Ph.D. student in Engineering Management and Systems Engineering, Old Dominion University, USA

(e-mail: hfakh001@odu.edu) 
aspects will receive acclaim as having been managed effectively.

Understanding the management of the engineering design cycle is at the center of delivering successful engineering projects. There are five core areas in the engineering design cycle that need to be managed effectively for the delivery of successful products. These engineering stages include problem investigation, solution design, design validation, design implementation, and finally, implementation evaluation. The requirements engineering phase of engineering design is the most important in managing the success of projects since it defines the scope of engineering projects and thus guides project implementation and the overall project quality. To exhaustively document project requirements, there is a need to utilize various engineering tools and techniques such as natural language, use case diagrams, feature diagrams, problem frame diagrams, scenarios, and state machines. The use of video animations, simulations, and mobile devices can also help to elicit more elaborate user requirements in terms of both usabilities as well as quality. Also, we know that there is a need to focus on all the kinds of user requirements during requirements engineering, including project aspects, functional requirements, and quality attributes. There is also a need to focus on the quality criteria for the requirements to ensure that engineering projects exhibit the highest level of quality. Also, the late incorporation of quality attributes in engineering projects leads to poor project designs. To capture all project requirements, it is recommended to use additional research methods, including simulation, modeling, action research, laboratory or field experiments, case studies, and pilot projects.

\section{RESEARCH METHODS}

The inductive research approach is a theory-building process that seeks to use available observations to form generalizations that can then be tested for confirmation. The inductive research approach uses reasoning that moves from observation, pattern recognition, tentative hypotheses, and finally, theory development. The inductive research approach is sometimes referred to as the "bottom-up" approach because it starts with observations before formulating theories. It is worth noting that although the qualitative research approach is common, and the data used in such research is readily available, the means to evaluate such data to gain a deeper understanding is usually scarce. The inductive research approach thus steps in to fill this gap by offering various strategies for evaluating and understanding relationships in qualitative data. It is worth noting that induction is simply the drawing of themes, concepts, relationships, and models from raw data. This needs to be done by experienced and skilled researchers or evaluators [6]. The rationale for using the inductive approach in research is to enable the elicitation of the dominant, frequent, or significant themes present in raw data.

According to [7], the core purpose of the induction research process includes simplifying available data into short formats, outlining the underlying relationship and links between the data findings and research objectives, and finally formulating a framework or theory that interprets the underlying relationship between the variables in the data. The inductive process offers a reliable set of principles for use in evaluating qualitative data for drawing inferences of the relationship between variables. It is worth noting that the induction approach is usually straightforward and helps in generating a tentative understanding of the relationship between variables. In comparison to the deductive and abductive approaches, it is worth noting that the inductive approach is less complicated. The inductive research approach is also considered a "goal-free" approach since researchers are concerned with the actual program effect.

The general approach to an inductive research strategy includes first reading the data findings to identify the categories and themes. The development of the themes was a result of the recurrent evaluation of the data and understanding of the relationship in the data findings. The themes identification process is usually recurrent to ensure that all themes pertinent to the available data have been ironed out. This was followed by systematic coding of the themes to merge related themes or data, as well as evaluating the similarities and differences. It is worth noting that the inductive research process is consistent with the qualitative research processes, including data reduction, data display, and conclusion drawing.

It is worth noting that the induction process is similar to pattern coding but has some differences. One core difference is that the inductive coding approach may lack the identification of explanations, causes, or relationships among people. It is worth noting that the outcome of the inductive coding process is determined by the experience and skills of the evaluators. The inductive process has several key features used in the coding of data findings.

These features include category label, which is a word or short phrase for a category; category description, which is a description of the meaning of the category; text or data, which are the coded data in the categories; links, which are the relations between categories; and type of model or category, which is a category system.

The deductive research approach is a theory-testing process that starts with a known theory or known generalization and uses the available knowledge to evaluate if the theory can be extended to other specific instances. Also, the deductive research approach utilizes a quantitative research methodology and mainly uses the case study approach to formulate tentative hypotheses. According to [8], the deductive research approach uses reasoning that moves from theory, hypothesis, observation, and finally confirmation. The deductive research approach is also called the "top-down" approach because it begins with theory identification before moving to observations [9].

When used in the qualitative research methodology, the deductive approach ensures that the outcome of qualitative research is verified and thus assures readers of conviction on the findings of the research. According to Thomas (2006), deduction is considered the dominant approach of conducting research and helps in formulating reliable research findings. There is a need to depend on innate ideas related to existing knowledge and theories to develop new hypotheses and opinions that guide the development of new knowledge. It is worth noting that deductive research can begin from any theoretical base, either new or existing bases, and enable in 
development of newer theories.

There is a need to use detailed justifications of selected theoretical understandings and positions to ensure that the outcomes of the deductive process are reliable. It is worth noting that deductive research approaches are non-validated because they are dependent on theoretical positions and not observable facts. They are thus vulnerable to potential criticisms because they might be subjective. As such, other researchers might propose different theories and observations. Overall, deductive research approaches are more detailed, and they might effectively guide the development of new theories and knowledge bases.

According to [10], abduction is a research method and approach that seeks to fill the gap in understanding puzzling, ambiguous, and surprising phenomena. It helps in finding meaningful explanations and solutions to pending issues and beliefs that seem out of check. As such, an abduction is a complex form of research approach and reasoning. The abduction helps to fill the gap that is left when independently utilizing inductive and deductive research approaches. It fills the weaknesses left when using inductive and deductive research. It follows a pragmatist approach and can utilize messy and unpredictable observations. This is mainly because abduction can venture into questioning even wellknown knowledge areas as a means of advancing or restructuring knowledge.

The abduction is used to help in solving issues that cannot be solved by any of the existing theories or knowledge base, especially knowledge available when using deductive and inductive approaches. Also, The Author indicates that abduction involves the setting of hypotheses and selecting the best among them since it is usually a tentative approach and put forward a series of steps used for abductive reasoning, including detection of phenomena, detecting causal mechanisms, developing a causal model, evaluation of the causal model, and finally formulating the case. From this account of abduction, it is evident that the development and evaluation of hypotheses are complementary steps that need to work together for an effective abduction approach. Abduction is an exploratory approach to research and not confirmative. It is worth noting that deduction is the confirmative research approach due to its use of quantitative research.

They also present that abduction can consist of four major phases, including exploration, examination, selection, and explanation. The process of developing hypotheses is based on six core steps, including situation observation, research question development, research question evaluation, representation of experience phenomena, developing representation, and finally, creation of hypotheses. The use of this approach in formulating hypotheses helps in ensuring that the newfound issue is worth investigating and would be beneficial for society.

The abduction is related to deduction in that they are both approaches to understanding the concepts forming phenomena. Mitchell (2018) adds that abduction is usually a continuous research approach that utilizes pragmatism and a mix of different research methods and strategies to gain an understanding of phenomena. Abduction helps in questioning existing theories, correcting them, as well as developing superior theories. It is thus a knowledge acquisition and knowledge building theory and outlines that abduction can also be termed as a knowledge-extending approach because its focus is on evaluating available knowledge as well as formulating new hypotheses for testing. Also, it adds that abduction's main purpose is to explore the available data, find the possible patterns in the data, and suggest hypotheses based on the available categories. As such, abduction helps to clarify knowledge areas and restructure knowledge to remove ambiguity and overlapping aspects. It helps to question both known and unknown phenomena as a means of classifying and restructuring knowledge areas.

According to [11], the synthetic research approach emphasizes the reconstruction of dispatched units of an event or unit of study and summarizes each unit independently. The researcher should know the operation before starting reconstruction. The main aim of using this type of research is whenever the researchers want to develop a process from an abstract idea to a concrete knowledge construction while bringing together the constructs of an event of interest and understanding them. This type of approach utilizes a previous analysis of a phenomenon or an event to achieve the synthesis. Despite the previous analysis, an exhaustive understanding is drawn from further examining the constructs of a specific phenomenon. This means that there is a thorough analysis of all elements, inclusive of those that were previously isolated, to form a concrete understanding.

The synthetic research approach comes with specific rules that govern how the analysis should be constructed [12]. The first rule specifies that; the researcher should clarify the proposals, notions, and statements before searching for any notion of each component to be analyzed. The second rule states that; even though the analysis and the synthesis of an event differ strikingly, each of them acts as a complement for the other as both are dependent on each other. The third rule states that all obstacles and difficulties should be separated and examined differently for them to be clearly understood. From a general spectrum, this type of reach approach utilizes the basic and simple data already known in the reconstruction of a full complex understanding. The reason why the synthetic approach should be implemented in system engineering is that it brings together all pieces of data, synthesizes and analyzes them independently then brings a clear understanding as a whole. This method is suitable in the quality assurance realm as it brings together all misunderstandings and pieces of data of a project and analyzes them differently in the process of coming up with a solid understanding.

According to [13], scientific research involves the use of scientific verification instruments to validate the existence of a certain factor. Project management and quality assurance is the key to conducting a successful project. Also, it emphasizes the use of various experiments to evaluate the importance of appropriate project management as well as quality assurance. This is a vital research approach as it brings out the reason why certain projects do not perform to the required standards that are required by the organization initiating the whole project. The Author [14], [15] also explains the importance of this method as a way in which system engineers prove the data that they obtain from the observation that they have made. The researcher will, therefore, prove the hypothesis that was made at the 
beginning of the research.

This scientific method is vital as it drives innovation, and therefore quality assurance will be brought out clearly when dealing with either huge or small projects. Nevertheless, this research method is valuable when evaluating the importance of effective project management and quality assurance as it scientifically demonstrates how this happens therefore dispelling any hits or beliefs that someone has about false claims or weak research.

\section{THEORETICAL UNDERPINNING}

This theory is concerned with how data is conceptualized. The theory was developed by two American sociologists, namely Anselm Strauss and Barney Glaser. This theory perfectly suits the Inductive research method as it focuses on creating new knowledge from the analytics of diverse data. This theory states that a researcher should go to the field open-minded, unbiased, and with no preconceived ideologies as he is intended to derive the problems in question from the setting that will eventually lead to new knowledge creation. This theory is related to system engineering research as this field is a diverse field that should be approached with a clear mind ready to collect data that will aid in the construction of new knowledge.

This is the latest version of grounded theory. The theory explains that theories and data are not discovered, but they are formulated by real scientists who visit the scope of the research and implement control measures to help them gather new knowledge. This theory is common with the pragmatists and the constructivists from their nature of gathering and measuring construct models for the development of scientific knowledge. The theory supports the research model of system engineering research, for it has multiple disciplines built from numerous foundations.

\section{ROLE OF QUALITY ASSURANCE THROUGHOUT THE PROJECT LIFE CYCLE}

Quality assurance plays a big role in guaranteeing the high quality of engineering projects and systems. Quality assurance refers to a discipline and a job that is focused on offering confidence and guaranteeing that a project will meet all quality aspects and requirements [16]. Quality assurance focuses on ensuring that a project or product meets both internal and external quality expectations. Internally, quality assurance ensures that a project meets organizational needs. Externally, quality assurance seeks to guarantee that a project will meet stakeholder quality expectations for regulators, government agencies, customers, and other stakeholders. Quality assurance is closely related to quality control, although the two terms have a slight difference in that quality control is a subset of quality assurance. Quality control refers to a set of mechanisms put in place to fulfill quality requirements. To fulfill these quality requirements, quality assurance utilizes a set of methods such as inspection and auditing. Inspection in quality assurance refers to the process of examining, measuring, and testing various project characteristics and aspects while comparing these with design specifications and requirements to ensure that there is conformity as well as guarantee that they meet the highest quality standards. The inspection might be concerned with examining products, modules, deliverables, processes, and other aspects of projects to ensure that they meet design specifications [17]. On the other hand, auditing is a quality assurance function concerned with evaluating the manifesting quality conditions of a project, comparing it with expectations, and preparing reports for reporting purposes. As such, auditing might use inspection in project evaluation, but it involves documentation of a detailed report indicating whether the project meets quality expectations and specifications.

According to [18], the ever-increasing complexity and market pressure in engineering projects have necessitated carrying out quality assurance throughout a project to guarantee high quality. Quality assurance is an elusive aspect of project management because of the various complex issues related to project management. Conducting quality assurance right from the requirements gathering stage of project development helps in the identification of quality issues right from the requirements engineering phase. Quality assurance from the beginning of a project helps in avoiding instances where project development is based on erroneous initial requirements. As such, quality assurance helps to reduce enormous rework tasks in case there exist errors in the initial design specifications. Quality assurance helps to reduce the errors and bugs that could be identified during the testing phase of project implementation. The documents that quality is an elusive aspect of defining because it is dependent on organizational objectives and goals, as well as stakeholder perspectives. In light of this, also present several quality views that need to be considered when working on quality assurance. The first quality view is called the transcendental view, which views quality as a complex ideal that can never be achieved and sustained. The second quality view is called the user view and evaluates the quality of a project based on its ability to meet user expectations and requirements. The third quality view is called the manufacturing view, which focuses on the quality of a project during development as well as after delivery. The fourth quality view is called the product view and focuses on the internal and external aspects of a project. This view focuses on the use of the product based on internal and external capability. The final view is called the value-based view, which focuses on relating quality to cost. This view focuses on quality as a virtue that can be purchased and evaluates the usefulness of the quality of a project. Adhering to these quality views helps to visualize the required stakeholder input in the quality assurance process, thus guaranteeing the success of the process.

The authors also put forward various quality attributes that need to be met when working on the quality assurance process. The first quality attribute is correctness, which focuses on ensuring that the intended user requirements meet user and customer needs. Another quality attribute that needs to be met is unambiguity. Unambiguity refers to the exactness and aptness of requirements and specifications in that they can only be defined using one interpretation.

As such, a user specification or quality aspect must maintain the same definition for all the stakeholders using the system. Another important quality attribute is completeness, which focuses on ensuring that all elements needed to fulfill 
a certain quality requirement for a stakeholder are met. As such, project modules should not perform some tasks while failing to perform others. Consistency is another core quality attribute and focuses on ensuring that a specific requirement is consistent with other requirements as well as needed constraints.

The paper also put forward verifiability as another core quality aspect. Verifiability focuses on ensuring that all modules and project requirements are verifiable. As such, there must exist criteria for verifying that the requirement is met or not. Stability is another quality aspect and focuses on ensuring that project requirements are consistent and helpful in a project even after the project or requirements change. Another important quality aspect is modifiability and focuses on ensuring that all user requirements can be modified or scalable. Requirements must also be feasible, which means that there must exist human or technological means of accomplishing them such that the project achieves the set requirements. The right level of detail is another quality aspect and focuses on ensuring that the details available for each user requirement are sufficient to enable modification as well as understanding how the project executes the requirement. This quality aspect is closely related to comprehensibility, which focuses on ensuring that the requirements are phrased in a way that can be understood for modification or integration. Traceability is the last quality aspect and focuses on ensuring that design requirements can be traced throughout project development.

They also emphasize the need to use a quality strategy to ensure that quality assurance processes and activities are performed effectively. A quality strategy is a systematic approach that defines and specifies when, how, and where quality assurance techniques and approaches will be used to improve quality. When focusing on the utilization of a quality strategy, various aspects can be used to guide quality assurance implementation. The core aspects that need to be evaluated when implementing a quality strategy include the quality of user requirements, available resources, the available schedule, the risks involved, and the organization's aspects.

\section{CONCLUSION}

In conclusion, quality management, which entails quality assurance and quality control, is a challenging endeavor in any engineering project. This is because of the complexity of quality aspects as well as the unstable definition of quality for each of the project stakeholders. As such, quality management is a continuous and ever-changing aspect of project management and requires the effective utilization of quality tools, techniques, and models to succeed. It is important to conduct quality assurance right from the requirements engineering phase of project development to ensure that there are reduced errors and challenges during project implementation. There is a need to incorporate feedback and input from various project stakeholders to guarantee the achievement of high-quality standards and aspects. The complexity of project quality management requires input from various project development stakeholders to visualize and understand areas of concern.

\section{REFERENCES}

[1] Wieringa, R., \& Heerkens, H., "Designing requirements engineering research," Fifth International Workshop on Comparative Evaluation in Requirements Engineering, pp. 36-48, 2007.

[2] Al-Hajj, A., \& Zraunig, M., "The impact of project management implementation on the successful completion of projects in construction," International Journal of Innovation, Management, and Technology, 9(1), 21-27, 2018.

[3] Hyde, K. F., "Recognizing deductive processes in qualitative research," Qualitative Market Research: An International Journal, 2000.

[4] Radujković, M., \& Sjekavica, M., "Project management success factors. Procedia Engineering," vol. 196, pp. 607-615, 2017.

[5] Tahami, H., Mirzazadeh, A., Arshadi-khamseh, A., \& GholamiQadikolaei, A., "A periodic review integrated inventory model for buyer's unidentified protection interval demand distribution," Cogent Engineering, 3(1), 1206689, 2016.

[6] Fakhravar, H., Quantifying Uncertainty in Risk Assessment using Fuzzy Theory. ArXiv E-Prints, arXiv:2009.09334, 2020.

[7] Thomas, D. R., "A general inductive approach for analyzing qualitative evaluation data," American Journal of Evaluation, vol. 27(2), pp. 237246, 2006.

[8] Mitchell, A., "A review of mixed methods, pragmatism and abduction techniques," Proceedings of the European Conference on Research Methods for Business \& Management Studies, pp. 269-277, 2018.

[9] Tahami, H., \& Fakhravar, H., "A Fuzzy Inventory Model Considering Imperfect Quality Items with Receiving Reparative Batch and Order," European Journal of Engineering and Technology Research, vol. 5(10), pp. 1179-1185, 2020.

[10] Żelechowska, D., Żyluk, N., \& Urbański, M., "Find out a new method to study abductive reasoning in empirical research," International Journal of Qualitative Methods, 19, 1609406920909674, 2020.

[11] Williams, C., "Research methods," Journal of Business \& Economics Research (JBER), 5(3), 2007.

[12] Chapman, S., McNeill, P., \& Mcneill, P., Research methods. Routledge, 2005.

[13] Karasar, N., Scientific research method: concepts, principles, techniques. Ankara: 3A Ara, 2017.

[14] Tahami, H., Mirzazadeh, A., \& Gholami-Qadikolaei, A., "Simultaneous control on lead time elements and ordering cost for an inflationary inventory-production model with mixture of normal distributions LTD under finite capacity," RAIRO-Operations Research, vol. 53(4), pp. 1357-1384, 2019.

[15] Taghizadeh, E. (n.d.). Operations Management within Sharing Economic Industries,

[16] Eder, W. E., "Engineering Design: Role of Theory, Models, and Methods. In An Anthology of Theories and Models of Design", Springer. pp. 197-217, 2014.

[17] Tahami, H., \& Fakhravar, H., "Multilevel Reorder Strategy-based Supply Chain Model," 5th North American Conference on Industrial Engineering and Operations Management (IEOM), Michigan, USA, $2020 \mathrm{~b}$.

[18] Denger, C., \& Olsson, T., "Quality assurance in requirements engineering. In Engineering and managing software requirements, Springer, pp. 163-185, 2005.

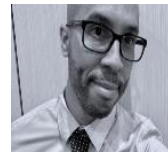

Monier Madison Ouabira is a Ph.D. student in Engineering Management and Systems Engineering at Old Dominion University. He received His M.S. degree in Project Management. $\mathrm{He}$ is working as a Senior Engineering Manager/ Program Manager at Massachusetts Institute of Technology (MIT) in Boston, MA. USA.

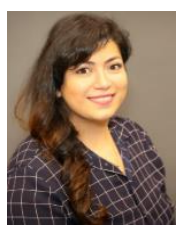

Hengameh Fakhravar is a Ph.D. student in Engineering Management and Systems Engineering at Old Dominion University. She received her M.S. degree in Industrial Engineering. She is working as a Graduate Assistant in the Engineering Management and Systems Engineering Department. Her research interests are Statistical analysis, Fuzzy methods, System engineering. 